\title{
Casein micelle dissociation in skim milk during high-pressure treatment: Effects of pressure, $\mathrm{pH}$, and temperature
}

\author{
V. Orlien, ${ }^{1}$ L. Boserup, and K. Olsen \\ Food Chemistry, Department of Food Science, Faculty of Life Sciences, University of Copenhagen, Rolighedsvej 30, DK-1958 Frederiksberg C, \\ Denmark
}

\begin{abstract}
The effect of $\mathrm{pH}$ (from 5.5 to 7.5 ) and temperature (from 5 to $40^{\circ} \mathrm{C}$ ) on the turbidity of reconstituted skim milk powder was investigated at ambient pressure and in situ under pressure (up to $500 \mathrm{MPa}$ ) by measurement of light scattering. High-pressure treatment reduced the turbidity of milk for all combinations of $\mathrm{pH}$ and temperature due to micelle dissociation. The turbidity profiles had a characteristic sigmoidal shape in which almost no effect on turbidity was observed at low pressures (100 $\mathrm{MPa}$ ), followed by a stronger pressure dependency over a pressure range of $150 \mathrm{MPa}$ during which turbidity decreased extremely. From the turbidity profiles, the threshold pressure for disruption of micelle integrity was determined and ranged from $150 \mathrm{MPa}$ at low $\mathrm{pH}$ to 350-400 MPa at high $\mathrm{pH}$. The threshold pressure diagram clearly showed a relationship between the barostability of casein micelles and $\mathrm{pH}$, whereas almost no effect of temperature was shown. This remarkable $\mathrm{pH}$ effect was a consequence of pressure-induced changes in the electrostatic interactions between colloidal calcium phosphate and the caseins responsible for maintaining micellar structure and was explained by a shift in the calcium phosphate balance in the micelle-serum system. Accordingly, a mechanism for high pressure-induced disruption of micelle integrity is suggested in which the state of calcium plays a crucial role in the micelle dissociation process.
\end{abstract}

Key words: casein micelle, high pressure, dissociation, intramicellar interaction

\section{INTRODUCTION}

The integrity of casein micelles in milk is stabilized by hydrophobic interactions and electrostatic interactions, mainly through micellar clusters of calcium phosphate. It is known that high-pressure (HP) treatment of milk destabilizes the casein micelles, as shown by a more

Received March 26, 2009.

Accepted October 6, 2009.

${ }^{1}$ Corresponding author: vor@life.ku.dk translucent appearance, decreased turbidity, and a reduction in average casein micelle diameter (Gaucheron et al., 1997; Needs et al., 2000; Huppertz et al., 2004; Regnault et al., 2004; Anema et al., 2005; Orlien et al., 2006). The hydrophobic and electrostatic interactions are optimally balanced in native casein micelles, but the HP-induced changes of casein micelles suggest disruptive effects on the attractive and repulsive forces in the casein micelle structure under pressure. The findings of HP-induced micelle dissociation in skim milk at natural $\mathrm{pH}$ at pressures greater than intermediate pressures (200-300 MPa) together with an increase in released calcium and a marked hydration of the micelle suggest an interesting pressure dependency on the essential interactions and the role of water and calcium (Gaucheron et al., 1997; López-Fandiño et al., 1998; Huppertz et al., 2004; Regnault et al., 2004; Orlien et al., 2006; Anema, 2008); however, the mechanisms of micelle dissociation are not established. The dissolution of colloidal calcium phosphate (CCP) and the diminished strength of hydrophobic binding affect the disruption of casein micelles differently according to the initial state of the micelle integrity being dependent on temperature and $\mathrm{pH}$. Consequently, depending on the extent of disruption, different structures of HP-modified micelles are obtained, resulting in different functional properties of the caseins. Formation of HP-induced gels requires high casein concentrations and pressures above $400 \mathrm{MPa}$ to release the caseins from the micelles during pressure treatment with subsequent aggregation and gelation. Also, caseins may act as structural units in encapsulation of functional components such as vitamins and antioxidants, which are unaffected by pressure. In this way, casein micelles could be enriched with important nutraceuticals or antioxidants and deliver health-promoting nutrients or oxidative protection, respectively. To control the disruptive state of the HP-modified micelles, detailed investigations of the HP effects on the colloidal interactions in casein micelles were performed by measuring the turbidity of skim milk in situ under pressure over a broad range of temperature and $\mathrm{pH}$ values. 
MATERIALS AND METHODS

\section{Materials}

Low-heat skim milk powder, Milex 240, was obtained from Arla Foods (Akafa, Svenstrup, Denmark). Sodium azide and trypsin inhibitor were obtained from Sigma-Aldrich Chemie GmbH (Steinheim, Germany). Glucono- $\delta$-lactone, purity of $99 \%$, was obtained from Acros Organics (Morris Plains, NJ). Sodium hydroxide $(2.0 \mathrm{M})$ was of analytical grade, and all aqueous solutions were prepared in highly purified water (Milli-Q Plus, Millipore Corporation, Bedford, MA).

\section{Milk Sample Preparation}

Skim milk was prepared by dissolving skim milk powder in water to a final content of $100 \mathrm{~g} / \mathrm{L}$. Sodium azide $(0.10 \mathrm{~g} / \mathrm{L})$ and trypsin inhibitor $(0.01 \mathrm{~g} / \mathrm{L})$ were added. The reconstituted skim milk was equilibrated overnight at $20^{\circ} \mathrm{C}$ and kept at this temperature for no longer than $3 \mathrm{~d}$ until further treatment or measurement. The $\mathrm{pH}$ of milk samples was adjusted before the optical measurements of turbidity to 7.0 or 7.5 using $2.0 \mathrm{M} \mathrm{NaOH}$ or to 5.5 or 6.0 by adding 5.2 or $3.0 \mathrm{~g} / \mathrm{L}$ glucono- $\delta$-lactone, respectively, to the milk, and the samples were equilibrated overnight at $20^{\circ} \mathrm{C}$.

\section{In Situ Turbidimetry}

The intensity of light transmitted through the milk samples under pressure was measured in a temperaturecontrolled, HP optical cell (SITEC Sieber Engineering AG, Maur-Zürich, Switzerland) equipped with a handoperated, pressure-generating system and a combined deuterium-halogen light source (Hamamatsu Photonics K.K., Hamamatsu City, Shizuoka Pref., Japan). The HP optical cell had an optical pathway of $2 \mathrm{~mm}$, which allowed observation of highly turbid milk samples without dilution. The pressure-generating system was filled with the milk sample at a given $\mathrm{pH}(5.5,6.0$, $6.7,7.0$, or 7.5 ) or water and kept in the HP optical cell for $20 \mathrm{~min}$ to reach the respective temperatures $\left(5,10,20,30\right.$, or $\left.40^{\circ} \mathrm{C}\right)$. The intensity of transmitted light from 350 to $700 \mathrm{~nm}$ was recorded via a fiberoptic cable connected to an AVS-S2000 spectrometer (Avantes, Eerbeek, the Netherlands) and attached to a DAQ-700 A/D converter (Avantes) at pressures from 0.1 to $500 \mathrm{MPa}$ in steps of $50 \mathrm{MPa}$ (at each pressure, the intensity spectrum was measured when the actual pressure was reached within $1 \mathrm{~min}$ ). From the intensity, the turbidity $(\tau)$ was calculated using the following equation:

$$
\tau=-\frac{1}{0.2 \mathrm{~cm}} \ln \frac{\mathrm{I}}{\mathrm{I}^{\mathrm{ref}}},
$$

where I and $\mathrm{I}^{\text {ref }}$ are the intensities of transmitted light at a wavelength of $600 \mathrm{~nm}$ for the milk sample and for water as reference, respectively. The intensity of water was recorded before measurement of the milk sample. After each measurement, the system was cleaned. The presented turbidity values are the means and standard deviations of measurements on 3 to 5 different milk samples at the respective $\mathrm{pH}$ and temperature conditions.

\section{RESULTS AND DISCUSSION}

\section{Turbidimetry}

The casein micelles are composed of casein molecules, $\mathrm{CCP}$, and water molecules dispersed in the whey serum and are the main light-scattering particles in skim milk. Changes in $\mathrm{pH}$, temperature, and pressure can change the protein and mineral balance and the hydration level of the micelles; thus, turbidity measurement can be used to approximate changes in casein micelles, allowing a qualitative interpretation of the alteration in the micellar interactions. High-pressure treatment induces major changes in the turbidity of skim milk depending on both $\mathrm{pH}$ and temperature. Figure 1 shows the turbidity dependency on pressure, $\mathrm{pH}$, and temperature. As can be seen, the initial turbidity $\left(12.6 \leq \tau_{\mathrm{i}} \leq 28.5\right.$ at $0.1 \mathrm{MPa}$ ) varies considerably with temperature and $\mathrm{pH}$, whereas the final turbidity $\left(2.0 \leq \tau_{\mathrm{f}} \leq 6.1\right.$ at $\left.500 \mathrm{MPa}\right)$ is comparatively equal independent of temperature and $\mathrm{pH}$. It is emphasized, though, that the turbidity value represents the average light scattering of the solution, and all micelles might not scatter light equally. The turbidimetric technique is sensitive to multiple scattering and variations in the refractive index, and this has to be taken into account when turbidity is interpreted according to particle size distribution. The refractive index difference, when changing the mineral balance, the protein balance, and the hydration of micelles, is determined by the changes between that of micelles and of serum. The influence of changes in the refractive index on turbidity changes of milk has not been quantitatively established, but considering the small difference between that of water (1.333) and that of milk (1.338) (Walstra et al., 1999), the influence is assumed to have little effect. It is assumed that the factors responsible for changes in the micelle size under pressure are affecting the micelles similarly so that the average size is shifted to lesser values with the size dis- 

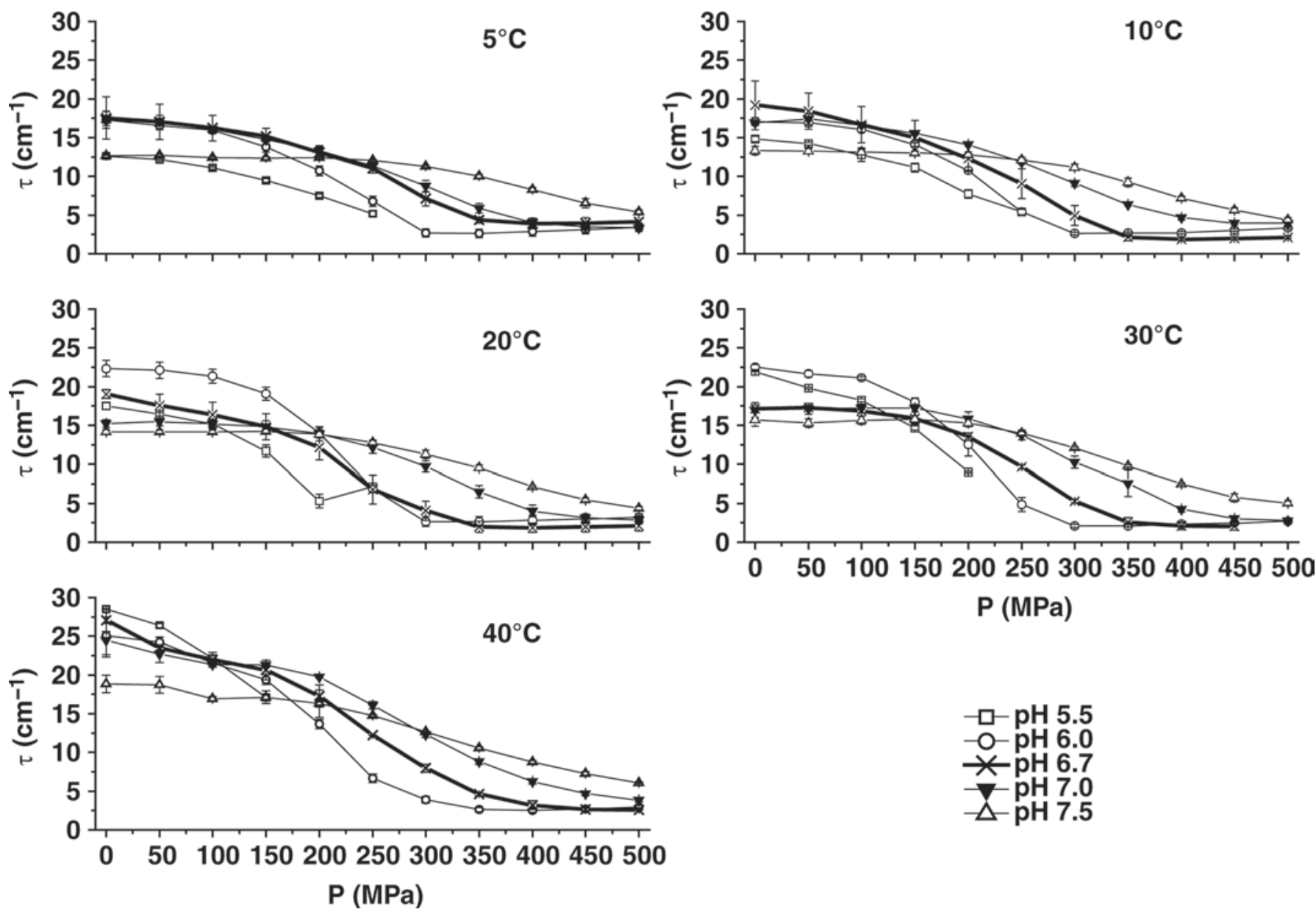

Figure 1. The in situ turbidity $(\tau)$ of reconstituted skim milk powder under pressure $(\mathrm{P})$ at temperatures from 5 to $40^{\circ} \mathrm{C}$ and $\mathrm{pH}$ from 5.5 to 7.5. The pressure was increased manually from 0.1 up to $500 \mathrm{MPa}$, and for every $50 \mathrm{MPa}$, an intensity spectrum of the milk was measured. The in situ turbidity was calculated using the intensity at $600 \mathrm{~nm}$. For the improvement of visual comparison, the lines connecting turbidity values of milk at natural $\mathrm{pH}$ are in bold. Error bars represent the SD of 3 to 5 individual milk samples.

tributed over some range, and any change in the light scattering (and turbidity) of milk reflects changes in the shape and composition of the micelles and, thus, in the colloidal interactions (Regnault et al., 2004; Anema et al., 2005; Gebhardt et al., 2005; Orlien et al., 2006). Currently, turbidimetry is the major practical method, albeit indirect, to monitor in situ micelle changes in milk during HP treatment.

\section{Turbidity of Milk as a Function of Temperature and $\mathrm{pH}$ at Ambient Pressure}

To establish the starting point of the micellar interactions, the individual effects of changing $\mathrm{pH}$ or temperature on the turbidity of milk were considered at ambient pressure before examining HP effects. It can be clearly seen in Figure 1 that $\mathrm{pH}$ and temperature had a considerable effect on micelle light scattering, and the initial turbidity levels were termed low at $\tau$
$=10-15$, intermediate at $\tau=15-20$, and high at $\tau=$ $20-30$.

Turbidity at Low Temperature. The casein micelles in milk at $\mathrm{pH} 5.5$ at $5^{\circ} \mathrm{C}$ had a loose structure resulting in minimum turbidity (Figure 1). The majority of the hydrophobic and electrostatic stabilizing interactions are lost because of increased solubility of $\mathrm{CCP}$, reduced charge of the caseins, and dissociation of the casein from the micelle (Rose, 1968; Roefs et al., 1985; Dalgleish and Law, 1988, 1989; Ono et al., 1990; Singh et al., 1996). At higher $\mathrm{pH}$, the more negative charge of the caseins and the lesser solubility of CCP facilitate increased electrostatic (casein/calcium) interactions and compensate for the low hydrophobic interactions at low temperature, resulting in a more dense structure and micelles that scatter light equally, with an intermediate turbidity at $\mathrm{pH} 6.0,6.7$, and 7.0 at $5^{\circ} \mathrm{C}$ (Figure 1). In accordance, an increase in micelle size has been observed when changing $\mathrm{pH}$ from acidic 
to neutral (Roefs et al., 1985; Anema et al., 2004; Donato et al., 2007), and an increase of the hydration of the micelles has also been observed (De La Fuente and Alais, 1975; Snoeren et al., 1984).

Increasing $\mathrm{pH}$ toward the alkaline side at $\mathrm{pH} 7.5$ will further increase the negative charge of the caseins, decrease the solubility of CCP, and lead to a looser expanded structure of the casein micelle. Consequently, electrostatic repulsion will disperse the charge in the micelles, resulting in sufficient permeability of water and, thus, increases in the hydration of the micelles and changes in the refractive index of the micelles toward that of water, as shown by low turbidity at $\mathrm{pH} 7.5$ and $5^{\circ} \mathrm{C}$ (Figure 1). Noticeably, milk samples with $\mathrm{pH} 5.5$ and 7.5 at $5^{\circ} \mathrm{C}$ have the same low turbidity, but it is unlikely that the composition of the micelles is similar because different intramicellar forces are responsible for maintaining the micelle integrity.

Turbidity at High Temperature. Currently, no relationship between high temperature and casein dissociation, CCP solubility, micelle hydration, micelle size, and turbidity in acidic and alkaline milk has been established. However, as shown in Figure 1, an increase in temperature enhanced the hydrophobic interactions, which contributed to stabilizing the micelle structure and resulted in an increase in turbidity for all $\mathrm{pH}$ values. Ono et al. (1990) reported an increase in turbidity of natural $\mathrm{pH}$ milk when changing the temperature from 4 to $37^{\circ} \mathrm{C}$ due to the association of smaller micelles. An increase in temperature was found to reduce the fractions of soluble caseins (Rose, 1968; Dalgleish and Law, 1988), the hydration of the micelles (Snoeren et al., 1984), and the solubility of CCP (Pouliot et al., 1989). In our experiment, the $\mathrm{pH}$ of the milk samples was adjusted at room temperature, allowing the $\mathrm{pH}$ effect to be established before the effect of temperature. Therefore, initially, some micelles were smaller at lower $\mathrm{pH}$ because of casein dissociation and dissolving of CCP, and some micelles swelled because of increased hydration. The subsequent increase in temperature up to $40^{\circ} \mathrm{C}$ effectively enhances the shift from dissolved caseins and calcium phosphate back to the micellar structure, and some smaller micelles might associate, resulting in larger micelles, with the greatest turbidity measured at $\mathrm{pH} 5.5,6.0,6.7$, and 7.0 at $40^{\circ} \mathrm{C}$ (Figure 1). However, in the alkaline region, the negatively charged caseins will attract water (favoring hydrogen bonding) to shield the charges, lowering the turbidity at $\mathrm{pH} 7.5$.

\section{In Situ Turbidity of Milk Under Pressure as a Function of $\mathrm{pH}$ and Temperature}

In Situ Turbidity. The general assumption is that a decrease in turbidity means that the micelle structure changes and most likely dissociates into smaller fragments. Hence, the observed decrease in the turbidity of milk during the stepwise increase in pressure from ambient pressure to $500 \mathrm{MPa}$ (Figure 1) indicates dissociation of the casein micelles. Overall, as was shown, low pressure $(100 \mathrm{MPa})$ resulted in a small decrease in the turbidity, indicating minor changes of the casein micelles. Except for the low-pH milk ( $\mathrm{pH} 5.5)$, for which the turbidity decreased initially upon pressurization and at pressure above $250 \mathrm{MPa}$ at $5^{\circ} \mathrm{C}$ and $150 \mathrm{MPa}$ at $40^{\circ} \mathrm{C}$, the intensity spectrum could not be measured because of precipitation of the caseins, which was probably a consequence of the HP-induced reduction of $\mathrm{pH}$ below the isoelectric point of the caseins. As the pressure increased past $100 \mathrm{MPa}$, the turbidity was markedly reduced, indicating that HP-treated milk changes from a turbid solution to a more clear solution. However, with high-pH milk ( $\mathrm{pH} 7.5$ ), almost no effect on turbidity was observed upon pressurization up to $200 \mathrm{MPa}$, indicating that some form of micellar structure was maintained.

For milk with $\mathrm{pH}$ above 5.5, there was a zone over a narrow pressure range in which important changes in the turbidity occurred. Notably, the zone occurred over a pressure range of approximately $150 \mathrm{MPa}$ for all the $\tau$ curves at the temperature and $\mathrm{pH}$ combinations investigated. The majority of the turbidity profiles were sigmoidal in nature, and as $\mathrm{pH}$ of the milk was increased, this zone was progressively shifted to greater pressures. Casein micelles are dynamic in nature, and with prolonged pressure time, micellar association becomes central compared with the initial HP-induced casein micelle dissociation (Orlien et al., 2006; Anema, 2008). To study the HP-induced micelle dissociation and to separate any micellar association phenomenon, the light transmission was measured immediately when the actual pressure was achieved (within $1 \mathrm{~min}$ ). Figure 1 shows that the observed zone displays a distinctive dependency on $\mathrm{pH}$ and temperature and indicates the importance of the internal (after the $\mathrm{pH}$ and temperature adjustment before HP treatment) micelle colloidal interactions on HP-induced micelle dissociation. The sigmoidal $\tau$ curve describes phenomena in which little change is apparent in response to pressure until a threshold that marks the onset of severe transformations into a new, stable, nonresponsive state is reached. Based on the sigmoidal nature, the data points were subjected to curve fitting based on the 4-parameter logistical equation (Figure 2):

$$
\tau(\mathrm{P})=\frac{\tau_{\mathrm{i}}-\tau_{\mathrm{f}}}{1+\left(\mathrm{P} / \mathrm{P}_{\mathrm{t}}\right)^{\mathrm{b}}}+\tau_{\mathrm{f}},
$$




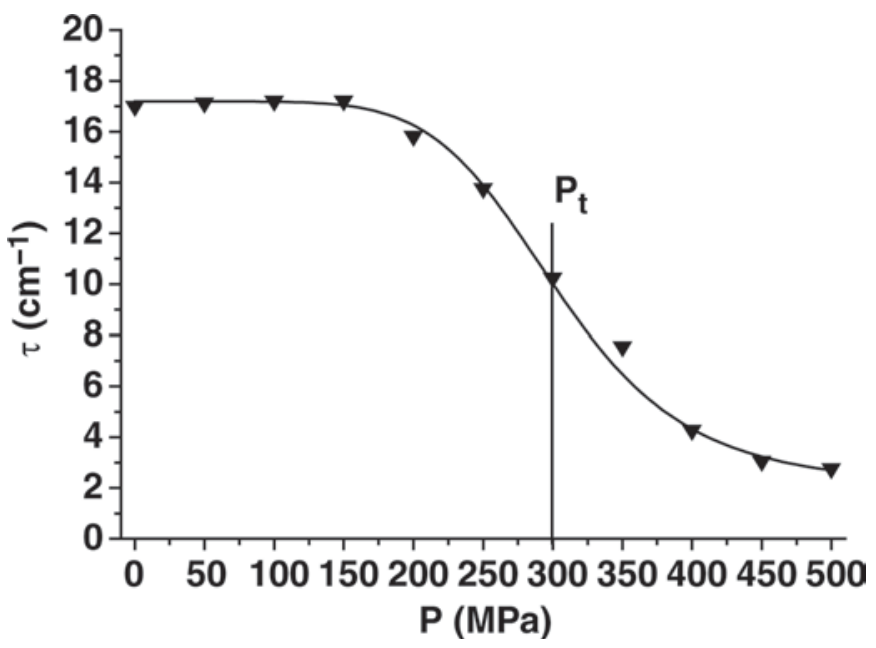

Figure 2. The logistical curve fitting based on Equation 2 of the measured in situ turbidity $(\tau)$ under pressure $(\mathrm{P})$ of reconstituted skim milk powder at $\mathrm{pH} 7.0$ and $30^{\circ} \mathrm{C}$ with the threshold pressure $\left(\mathrm{P}_{\mathrm{t}}\right)$ marked.

where $\tau(\mathrm{P})$ is the response of the turbidity at the applied pressure, $\mathrm{P} ; \tau_{\mathrm{i}}$ is the initial turbidity at $\mathrm{P}=0.1$ $\mathrm{MPa} ; \tau_{\mathrm{f}}$ is the final turbidity at $500 \mathrm{MPa} ; \mathrm{P}_{\mathrm{t}}$ is the threshold pressure halfway between the limiting values $\tau_{\mathrm{f}}$ and $\tau_{\mathrm{i}}$; and $\mathrm{b}$ is a slope factor that determines the steepness of the curve (depending on the cooperativity of the inter- and intramolecular interactions in the casein micelle around the actual threshold pressure). We interpreted $\mathrm{P}_{\mathrm{t}}$ as the threshold pressure for disruption of the micelle integrity maintained at initial conditions and subsequent dissociation into some type of micelle fragments still capable of scattering light. Using the threshold pressure, we can deduce one pressure value from each turbidity curve and present the obtained threshold pressures in a 3-dimensional graph (Figure 3) that allows improved visualization of the effects of $\mathrm{pH}$ and temperature on casein micelle dissociation in reconstituted skim milk powder under pressure.

$\boldsymbol{P}_{\mathrm{t}}$ Dependency of $\boldsymbol{p H}$ and Temperature. It is apparent from Figure 3 that temperature and $\mathrm{pH}$ affect the barostability of the casein micelles differently, reflecting a change in the balance between intramicellar hydrophobic interactions and the attractive and repulsive electrostatic interactions as controlled by the charge of the caseins and the current state of CCP. During the initial pressure build-up, the incorporation of water molecules into the micellar structure destabilizes the hydrophobic contacts more easily relative to the electrostatic-based contacts (e.g., hydrogen-bonded water or $\mathrm{CCP}$ ), leading to disruption of the hydrophobic interactions analogous to the dissociation process of oligomeric proteins and denaturation of globular proteins (Weber, 1987). Following the HP-induced disruption of hydrophobic interactions, the dissolution of CCP into hydrated calcium phosphate and further dissociation into charged ions in serum is favored upon increased pressure due to electrostriction. The HP-induced dissociation of the phosphate salts and self-ionization of water in serum result in a $\mathrm{pH}$ decrease that favors dissolution of CCP and an increased concentration of phosphate ions that favors binding of calcium and, thus, affects the balance between CCP and hydrated calcium phosphate and dissociated ions. The mechanism, rate, and extent of the disruption of the interaction between caseins and CCP depend on the starting point of the micellar interactions in the initial state of the casein micelles. However, from Figure 3 it is clear that the threshold pressure depends to a greater degree on $\mathrm{pH}$ and less on temperature because HP disrupts hydrophobic interactions more easily than it does the stronger electrostatic interactions.

At natural milk $\mathrm{pH}$, there is a favorable balance of electrostatic interactions, and the threshold pressure around $250 \mathrm{MPa}$ indicates the early disruption of the important electrostatic attractive forces between CCP and the caseins. The solvation of the individual caseins and the contraction of water around the colloidal centers are promoted. This facilitates the dissociation of CCP due to ionic contraction of solvent water, increases the availability of calcium in the solution, and, finally, shields the intermolecular electrostatic repulsion promoting the pressure-induced solubilization of the caseins. Thus, the greatest possible pressure for

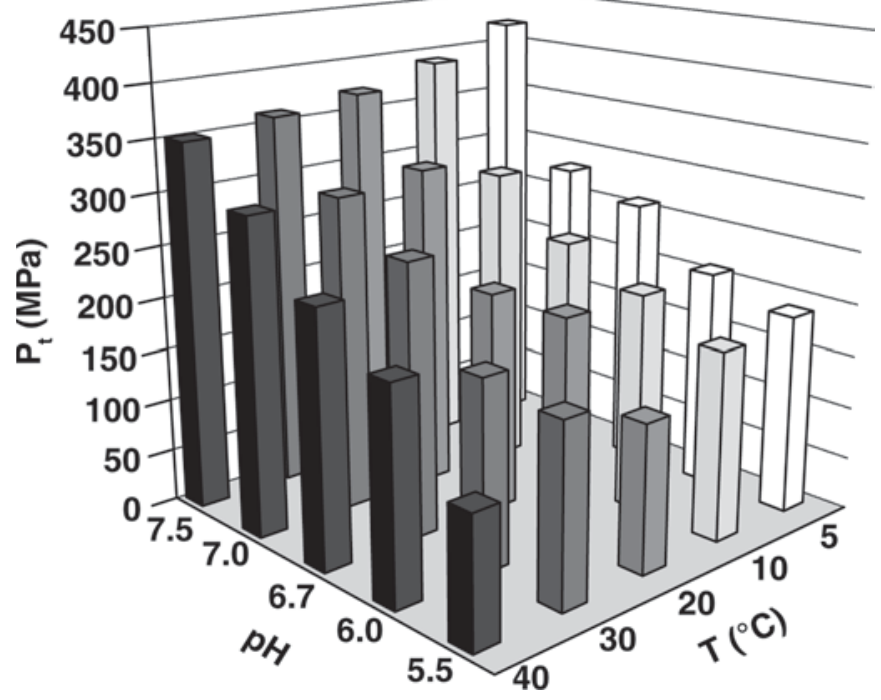

Figure 3. The barostability diagram of casein micelles in reconstituted skim milk powder. The threshold pressure $\left(\mathrm{P}_{\mathrm{t}}\right)$ for disruption of the casein micelle integrity in skim milk at temperatures (T) 5,10 , 20,30 , or $40^{\circ} \mathrm{C}$ and $\mathrm{pH} 5.5,6.0,6.7,7.0$, or 7.5 is presented. Data are means of 3 to 5 experiments on individual milk samples; the coefficient of variation was $<5 \%$ for each threshold level. 
maintaining the micellar stability at $\mathrm{pH} 6.0$ and 6.7 was around $250 \mathrm{MPa}$.

In acidified milk, the threshold pressure decreased (Figure 3) as a consequence of changed intramicellar interactions in the initial state of the micelles. The loose micelle structure due to increased level of hydration and dissolution of $\mathrm{CCP}$ and caseins at $\mathrm{pH}$ 5.5 rendered the micelles highly sensitive to HP. The initial micelle structure, which lacks most of the electrostatic stabilizing forces, favors the incorporation of water because the water-protein system may be packed more efficiently at relatively low pressure and, in turn, promotes further separation of CCP into hydrated calcium phosphate. Once the essential interactions are disrupted, an unstable structural arrangement is established at the threshold pressure in which micelle fragments are able to move independently. At pressures exceeding the threshold pressure, a more extensive or complete disruption of the micellar framework can take place, resulting in micelle dissociation at relatively low pressures.

Alkalization of milk had the opposite effect because strong electrostatic attractive forces dominate due to the negatively charged caseins and the increase in the level of CCP, thus stabilizing the micelles against HPinduced disruption. The more nonresponsive nature of the turbidity profiles (milk at $\mathrm{pH} 7.5$ in Figure 1) is a reflection of the enhanced capacity to maintain micellar integrity. The initial HP-induced water inclusion and disruption of hydrophobic interactions are rather difficult in the extensively hydrated micelles, and greater pressure is required before the hydration of the casein and CCP render the structure unstable. Moreover, the intramicellar interaction between the caseins and CCP is suggested to be superior in extent and seems to suppress the electrostrictive effect. Consequently, the dissolution of CCP into hydrated calcium phosphate occurred at a considerably greater threshold pressure (Figure 3), with subsequent dissociation of the micelle upon further pressurization.

A general feature for the casein micelles in all types of milk at pressures beyond the respective threshold pressures was complete dissociation. Because it is unlikely that there are similar structured micelle fragments in the vicinity of the threshold pressure in the different milk samples, it is most likely that there are very similar calcium-depleted, small micelle fragments at the greatest pressure as comparable low turbidity is measured at $500 \mathrm{MPa}$.

\section{CONCLUSIONS}

We have shown that HP induces marked changes to the micelle structure and have described the HP effects on the colloidal interactions important for micelle integrity over a broad range of temperature and $\mathrm{pH}$ values in reconstituted skim milk. The initial regulation of $\mathrm{pH}$ and temperature in skim milk at ambient pressure has different consequences on the intramicellar interactions. Thus, during HP treatment, the disruption of micelle integrity depends on different colloidal characteristics, and the mechanism of dissociation differs correspondingly. Overall, electrostatic interactions controlled the barostability of the casein micelles in skim milk and were explained by the greater importance of the state of calcium and the associated HP effect on the CCP balance in the micelle-serum system. The knowledge of the $\mathrm{pH}$ influence on the colloidal interactions can be used in the control of HP-modified micelle fragments. Different micellar fragments can be produced in the vicinity of the threshold pressure that differ in the electrostatic binding of the micellar framework, leading to functional units that may incorporate important compounds such as antioxidants and vitamins by exchange processes with CCP. The temperature independency of the threshold pressure ascertains that the formation of such functional units is not affected when the milk system is subjected to adiabatic heating during pressure build-up in industrial scale. High pressure in combination with $\mathrm{pH}$ management can be developed as novel methods for encapsulation, protection, and delivery of important nutrients in dairy systems.

\section{REFERENCES}

Anema, S. G. 2008. Effect of milk solids concentration on whey protein denaturation, particle size changes and solubilization of casein in high-pressure-treated skim milk. Int. Dairy J. 18:228-235.

Anema, S. G., E. K. Lowe, and K. S. Lee. 2004. Effect of pH at heating on the acid-induced aggregation of casein micelles in reconstituted skim milk. Lebensm. Wiss. Technol. 37:779-787.

Anema, S. G., E. K. Lowe, and R. Stockmann. 2005. Particle size changes and casein solubilisation in high-pressure-treated skim milk. Food Hydrocoll. 9:257-267.

Dalgleish, D. G., and A. J. R. Law. 1988. pH-Induced dissociation of bovine casein micelles. I. Analysis of liberated caseins. J. Dairy Res. 55:529-538.

Dalgleish, D. G., and A. J. R. Law. 1989. pH-Induced dissociation of bovine casein micelles. II. Mineral solubilization and its relation to casein release. J. Dairy Res. 56:727-735.

De La Fuente, B. T., and C. Alais. 1975. Solvation of casein in bovine milk. J. Dairy Sci. 58:293-300.

Donato, L., M. Alexander, and D. G. Dalgleish. 2007. Acid gelation in heated and unheated milks: Interactions between serum protein complexes and the surfaces of casein micelles. J. Agric. Food Chem. 55:4160-4168.

Gaucheron, F., M. H. Famelart, F. Mariette, K. Raulot, F. Michel, and Y. Le Graet. 1997. Combined effects of temperature and highpressure treatments on physicochemical characteristics of skim milk. Food Chem. 5:439-447.

Gebhardt, R., W. Doster, J. Friedrich, and U. Kulozik. 2005. Pressureinduced dissociation of casein micelles: Size distribution and effect of temperature. Braz. J. Med. Biol. Res. 38:1209-1214.

Huppertz, T., P. F. Fox, and A. L. Kelly. 2004. High pressure treatment of bovine milk: Effects on casein micelles and whey proteins. J. Dairy Res. 71:97-106. 
López-Fandiño, R., A. De La Fuente, M. Ramos, and A. Olano. 1998. Distribution of minerals and proteins between the soluble and colloidal phases of pressurized milks from different species. J. Dairy Res. 65:69-78.

Needs, E. C., R. A. Stenning, A. L. Gill, V. Ferragut, and G. T. Rich. 2000. High-pressure treatment of milk: Effects on casein micelle structure and on enzymic coagulation. J. Dairy Res. 67:31-42.

Ono, T., T. Murayama, S. Kaketa, and S. Odagiri. 1990. Changes in the protein composition and size distribution of bovine casein micelles induced by cooling. Agric. Biol. Chem. 54:1385-1392.

Orlien, V., J. C. Knudsen, M. Colon, and L. H. Skibsted. 2006 Dynamics of casein micelles in skim milk during and after high pressure treatment. Food Chem. 98:513-521.

Pouliot, Y., M. Boulet, and P. Paquin. 1989. Observations on the heat-induced salt balance changes in milk. I. Effect of heating time between 4 and $90{ }^{\circ} \mathrm{C}$. J. Dairy Res. 56:185-192.

Regnault, S., M. Thiebauld, E. Dumay, and J. C. Cheftel. 2004. Pressurisation of raw skim milk and of a dispersion of phosphocaseinate at $9{ }^{\circ} \mathrm{C}$ or $20{ }^{\circ} \mathrm{C}$ : Effects on casein micelle size distribution. Int. Dairy J. 14:55-68.
Roefs, S. P. F. M., P. Walstra, D. G. Dalgleish, and D. S. Horne 1985. Preliminary note on the change in casein micelles caused by acidification. Neth. Milk Dairy J. 39:119-122.

Rose, D. 1968. Relation between micellar and serum casein in bovine milk. J. Dairy Sci. 51:1897-1902.

Singh, H., M. S. Roberts, P. A. Munro, and C. T. Teo. 1996. Acidinduced dissociation of casein micelles in milk: Effects of heat treatment. J. Dairy Sci. 79:1340-1346.

Snoeren, T. H. M., H. J. Klok, A. C. M. Van Hooydonk, and A. J. Damman. 1984. The voluminosity of casein micelles. Milchwissenschaft 39:461-463.

Walstra, P., T. J. Geurts, A. Noomen, A. Jellema, and M. A. J. S. van Boekel. 1999. Dairy Technology. Principles of Milk Properties and Processes. Marcel Dekker, New York, NY.

Weber, G. 1987. Dissociation of oligomeric proteins by hydrostatic pressure. Pages 401-420 in High Pressure Chemistry and Biochemistry. R. van Eldik and J. Jonas, ed. Riedel Publishing Co., Dordrecht, the Netherlands. 